\title{
SIMULAÇÃO DE UM ESCOAMENTO BIFÁSICO ÓLEO- ÁGUA EM RESERVATÓRIO DE PETRÓLEO
}

\author{
T. B. FORTUNATO ${ }^{1}$, J. C. S. DUTRA ${ }^{2}$ e W. B. da SILVA ${ }^{3}$
}

\author{
LAMCES - Laboratório de Métodos Computacionais, Controle e Estimação \\ Universidade Federal do Espírito Santo - Engenharia Química (campus de Alegre) \\ ${ }^{1}$ tarsis.bf@hotmail.com; ${ }^{2}$ juliosdutra@yahoo.com.br; ${ }^{3}$ wellingtonuff@yahoo.com.br
}

RESUMO - Este trabalho aborda uma metodologia numérica para a simulação de escoamento bifásico imiscível em meios porosos no contexto de reservatório de petróleo para óleo e água. As Equações Diferenciais Parciais (EDP) de natureza acoplada que modelam o escoamento são manipuladas, de forma a se obter um sistema segregado de equações. A metodologia empregada faz uso de um esquema de solução que resolve o campo pressão implicitamente e o campo de saturação explicitamente, sendo que estes campos são acoplados por um termo de velocidade. Uma vez definidas as formulações matemáticas, o método de Volumes Finitos é utilizado para discretizar as equações. Com a implementação das rotinas computacionais em SCILAB, os resultados de simulação são consistentes e estão de acordo com a literatura.

\section{INTRODUÇÃO}

Os primeiros simuladores de reservatório de petróleo nasceram na década dos anos 50 do século passado, quando indústrias petroleiras concentraram esforços em pesquisa para acoplar análise numérica e os computadores da época. A motivação destas pesquisas continua sendo o planejamento das atividades de produção com base na projeção da simulação (Cordazzo, 2006). Esses simuladores utilizam modelos que são baseados na equação da continuidade e a lei de Darcy. Dentre os modelos conhecidos, uma modelagem bastante difundida é a de Peaceman (1977). Esse modelo permite o uso de métodos especializados capazes de explorar as particularidades matemáticas de cada equação, como a diferença na discretização em equações diferenciais parciais elípticas, hiperbólicas e parabólicas. Os modelos são formados por conjuntos de equações diferenciais parciais que, em sua maioria, não possuem solução analítica, sendo então solucionados por meio de métodos numéricos.

Os métodos numéricos utilizados para a resolução das equações que descrevem o escoamento em reservatórios geralmente são: Diferenças Finitas, Volumes Finitos e Elementos Finitos. Dentre estes métodos, a característica de conservação de grandezas físicas pelo método de Volumes Finitos o torna atrativo. Este método possui inúmeras variações, algumas delas são caracterizadas pela disposição espacial e quantidade de volumes de controles utilizados no balanço da grandeza. Em simulações de reservatório de petróleo, uma técnica que faz os balanços apenas entre dois volumes discretos, é conhecida como two point flux aproximation (TPFA) (Aarnes et al., 2007). A técnica TPFA é utilizada para discretização da equação difusiva da pressão. A equação da saturação, que tem caráter advectivo, é discretizada com a técnica upwind, a qual visa evitar o aparecimento de oscilações numéricas 
com uma fórmula sensível ao sentido de fluxo (Maslika, 2004). Ambas as técnicas, geralmente são utilizadas em malhas estruturadas.

Estes métodos numéricos são associados a diferentes esquemas de solução, que permitem explorar alguns fatores como discretização, implementação computacional e integração no tempo. Na simulação de reservatórios de petróleo, existem algumas variações destes esquemas, como as técnicas conhecidas como IMPES (implicit in pressure and explicit in saturation) e SS (solução simultânea). O IMPES é um esquema que tem o objetivo de obter uma melhor estabilidade, sem implicar em elevado custo computacional.

Neste contexto, o presente artigo aborda a simulação de escoamento em meio poroso, utilizando a técnica TPFA para discretizar os termos difusivos e a técnica upwind para discretizar os termos advectivos, ambos em malha estruturada. O esquema de solução adotado é o método IMPES, sendo que a simulação foi implementada no software SCILAB.

\section{FORMULAÇÃO MATEMÁTICA}

O modelo matemático, encontrado em Peaceman (1977), que descreve o escoamento imiscível de duas fases em reservatório de petróleo, é mostrado nas Equações 1 e 2. A Equação 1 é denominada Equação da Pressão, e a Equação 2 é denominada Equação da Saturação. Essa formulação é caracterizada como segregada, pois é obtido um sistema de equações que é resolvido da seguinte forma: primeiramente, o campo de pressão é solucionado e, posteriormente, o campo de saturação, sendo que esses dois campos são acoplados por meio de uma equação de velocidade.

$$
\begin{aligned}
& \nabla \cdot\left(-\lambda_{T} K \nabla p\right)=Q \\
& \phi \frac{\partial S_{w}}{\partial t}=-\nabla \cdot f_{w} v+Q_{w}
\end{aligned}
$$

Nestas representações, $\lambda_{T}$, é a mobilidade total, que é função das mobilidades das fases $\lambda_{i}$. O fluxo fracional da água é representado por $f_{w}$. A relação entre essas variáveis é descrita nas Equações 3 a 5. O termo K é a permeabilidade absoluta, $S_{w}$ a saturação da água, sendo que $S_{w}+S_{n}=1$. Este trabalho adotou o subscrito w para a fase molhante (água), e $\mathrm{n}$ para nãomolhante (óleo). Na Equação 1, $Q=Q_{w}+Q_{n}$ é injeção total.

$$
\begin{aligned}
& \lambda_{i}=\frac{k_{r i}}{\mu_{i}} \\
& \lambda_{T}=\lambda_{o}+\lambda_{w} \\
& f_{w}=\lambda_{w} / \lambda_{T}
\end{aligned}
$$

As permeabilidades relativas, ${ }_{r i}$ ' e as saturações das fases são relacionadas através das seguintes expressões encontradas em Carvalho (2005):

$$
k_{r w}\left(S_{w}\right)=\left(\frac{S_{w}-S_{r w}}{1-S_{r w}-S_{r n}}\right)^{2}
$$




$$
k_{r n}\left(S_{w}\right)=\left(\frac{1-S_{w}-S_{r w}}{1-S_{r w}-S_{r n}}\right)^{2}
$$

Nas Equações 6 e 7, $S_{r n}$ e $S_{r w}$ são as saturações residuais do óleo e água respectivamente. O termo de acoplamento utilizando a velocidade, é dado pela Equação 8:

$$
\nabla \cdot v=Q
$$

\section{FORMULAÇÃO NUMÉRICA}

Para a simulação de reservatórios de petróleo, é necessário um espaço físico para o escoamento ter sentido e, do mesmo modo, a criação de volumes discretos neste domínio para a aplicação do método de Volumes Finitos. Então, no desenvolvimento do método numérico, pode-se definir que o domínio $\Psi$ é composto por finitas células da malha denotadas por $\Psi_{i}$. (Figura 1). Ao se tratar da malha, as características dos fluídos e da rocha são de extrema importância. Na simulação numérica deste trabalho, utiliza-se uma abordagem macroscópica, onde as propriedades são consideradas contínuas em todo o domínio.

Figura 1 - Geometria da Malha
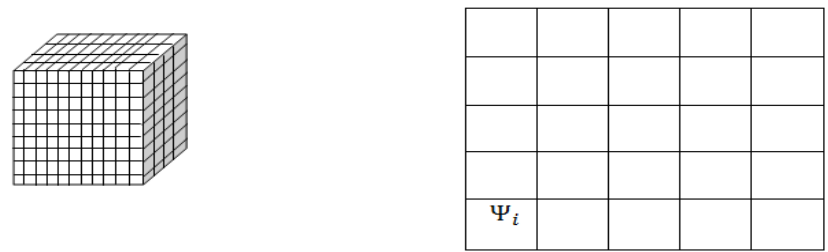

\subsection{Discretização da Equação da Pressão}

Para obter um conjunto de equações discretas que determinam o campo de pressão de um reservatório denotado por $\Psi$, com células de malha denominadas $\Psi_{i}$, aplica-se o método de Volumes Finitos TPFA na Equação 1. O resultado da discretização é mostrado na Equação 9. O termo de transmissibilidade, ${ }^{T}{ }_{i j}$ ' é dado pela Equação 10.

$$
\begin{aligned}
& \sum_{i} T_{i j}\left(p_{i}-p_{j}\right)=Q \\
& T_{i j}=2 K\left(\frac{d_{i}}{\lambda_{T i}}+\frac{d_{j}}{\lambda_{T j}}\right) A_{i j}
\end{aligned}
$$

$\mathrm{Na}$ Equação $10, A_{i j}$ é a área da interface entre a célula $\mathrm{i}$ e a célula $\mathrm{j}=\mathrm{i}+1, d_{i} \mathrm{e}^{d_{j}}$ representam a dimensão da coordenada discretizada no respectivo volume de controle. 


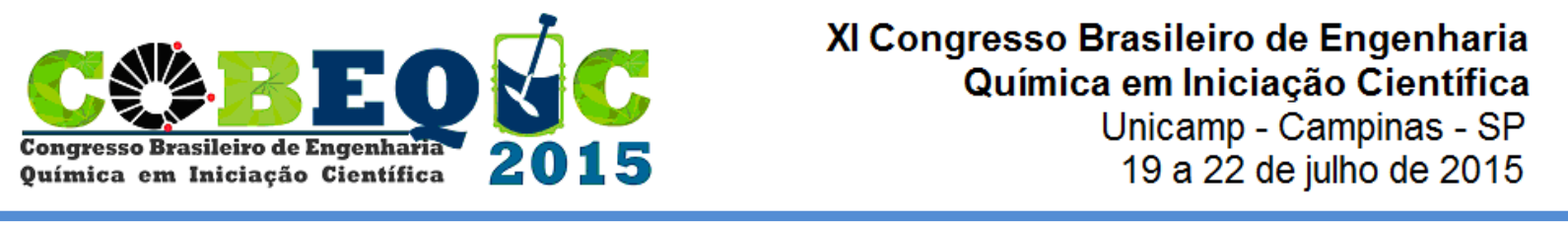

\subsection{Equação da Saturação}

Tomando a equação da Saturação, integrando no volume de cada célula da malha e utilizando a regra trapezoidal $(\theta$-rule) para a discretização temporal, obtém-se a discretização do campo de saturação:

$$
\frac{\phi}{\Delta t}\left(S_{w}^{k+1}-S_{w}^{k}\right)=-\frac{1}{\left|\Psi_{i}\right|} \sum\left(\theta F_{i j}^{k+1}+(1-\theta) F_{i j}^{k}\right)+Q_{w}
$$

onde o termo $F_{i j}^{k+1}=\int_{\Psi_{i}} f_{w} v_{i} \cdot n_{i j} \partial s$

Como se injeta somente água, Aarnes et al. (2007) propõem para o termo $Q_{w}$, a seguinte relação, $Q_{w}=Q^{+}+f_{w} Q^{-}$. Nesta representação, $Q^{+}$indica o fluxo que é injetado, e intuitivamente $Q^{-}$é o fluxo que deixa o volume de controle. Inserindo esta relação na Equação 20, rearranjando os termos, e fazendo $\theta=0$, obtém-se a Equação 21.

$$
\left.S_{w}^{k+1}=S_{w}^{k}+\phi\left|\Psi_{i}\right| Q^{+}-\sum_{j} F_{i j}^{k}+f_{w} Q^{-}\right)
$$

\section{ESQUEMA DE SOLUÇÃO: IMPES}

Este método objetiva uma melhor estabilidade da solução, sem implicar em elevado custo computacional. Porém, uma desvantagem está na solução explícita da Equação de Saturação. Essa solução é dependente do passo no tempo, de modo que uma escolha errada pode ocasionar oscilações e, até mesmo, não convergir. Em vista disto, deve ser estabelecida uma condição de estabilização. Aarnes et al. (2007) apresentaram uma condição de estabilização com dependência somente da velocidade. Dessa forma, a condição é atualizada somente mediante a um novo campo de pressão. A Equação 22 mostra a função deste passo no tempo para a solução da Equação de Saturação.

$$
\Delta t \leq \frac{\phi\left|\Psi_{i}\right|}{\max \left(Q^{+}\right)-\sum_{j} \min \left(v_{i j}, 0\right) \max :\left(f_{w}^{\prime}(s)\right)}
$$

onde o termo $f_{w}^{\prime}(s)=\frac{1}{1-S_{r w}-S_{r n} \partial k_{r w}}$

\section{EXEMPLO}

Este exemplo considera o escoamento bifásico imiscível óleo-água em um meio poroso homogêneo, isotrópico e bidimensional. Este problema foi adaptado de Carvalho (2005), cujo trabalho considerava um meio heterogêneo. Este exemplo é uma abordagem do clássico problema de $1 / 4$ de poço. O escoamento em meio poroso acontece em uma região de baixa permeabilidade entre o poço injetor e produtor. As saturações residuais foram consideradas como nulas. A viscosidade da água e óleo são, respectivamente, $\mu_{w}=0.001 \mathrm{~kg} / \mathrm{m} \cdot \mathrm{s}$ e $\mu_{n}=0.001 \mathrm{~kg} / \mathrm{m} \cdot \mathrm{s}$. A porosidade é constante em todo o domínio, $\phi=0.2$. A permeabilidade é 
um tensor diagonal, $K=10^{-7}[I] \mathrm{m}^{2}$. As condições de contorno nos poços injetor e produtor são $P_{\text {injetor }}=2 \cdot 10^{-7} \mathrm{~Pa}$ e $Q_{\text {produtor }}=-10.368 \mathrm{~m}^{3} /$ dia respectivamente. A geometria é um quadrado de lado igual 300 metros. A Figura 2 mostra a geometria adotada para o problema. A implementação do código para obtenção da simulação foi feita no software SCILAB. A malha foi discretizada com 300 células.

Figura 2 - Geometria do Problema.

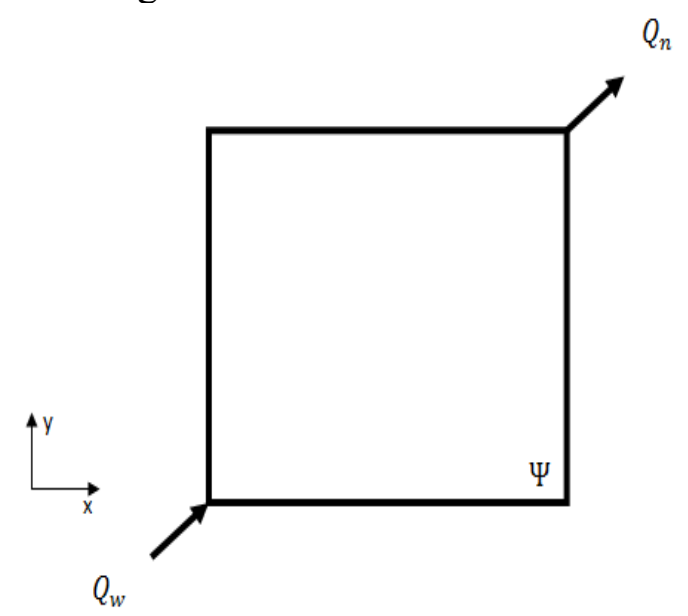

A Figura 3.a mostra o resultado do campo de pressão obtido com a técnica TPFA para um tempo 300 dias de produção, em uma malha estruturada com 300 células. A mesma configuração de tempo e células foi utilizada para obter o Perfil de Saturação mostrado na Figura 3.b.

Figura 3 - a) Campo de Pressão com tempo de simulação de 300 dias. b) Perfil de Saturação com tempo de simulação de 300 dias.

a)

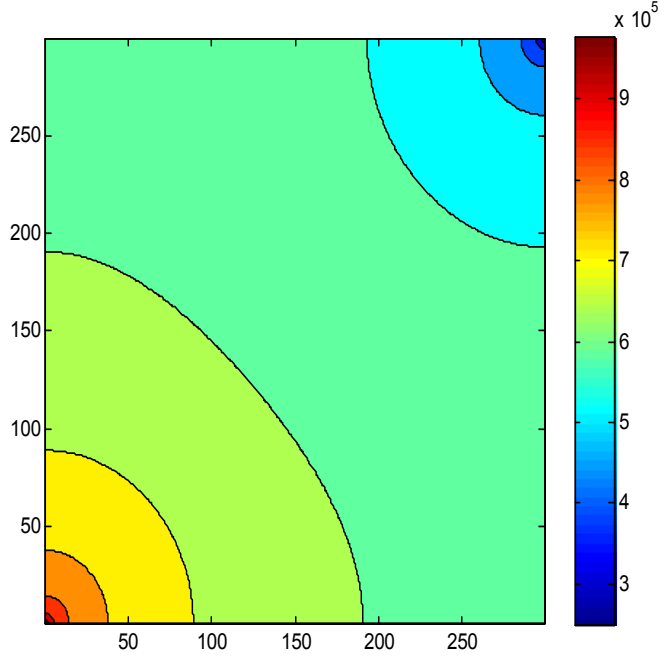

b)

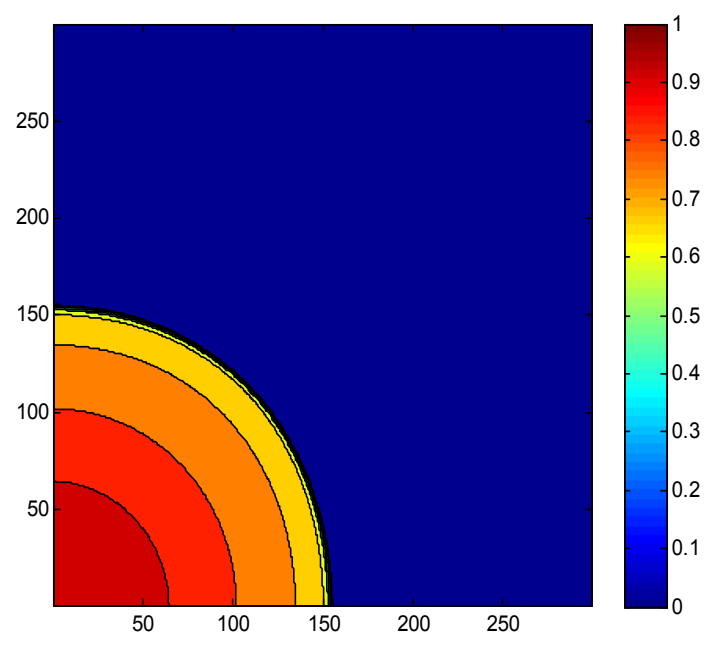

Para mostrar o desenvolvimento tanto do Campo de Pressão, quanto do Perfil de Saturação, foi simulado o comportamento de $1 / 4$ de poço para o tempo de 600 dias. $\mathrm{Na}$ Figura 4.a, percebe-se um pequeno aumento da pressão em algumas zonas. No Perfil de Saturação mostrado pela Figura 4.b, percebe-se que a frente de saturação perde o aspecto 


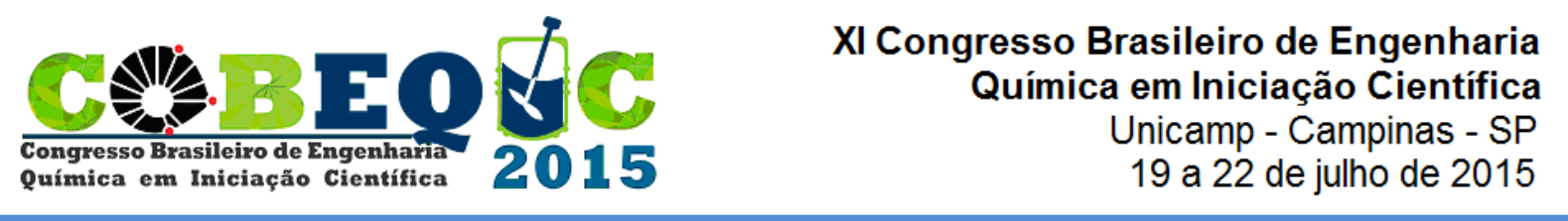

circular, tendendo a uma forma oval, que aponta para o poço de produção, além de um pequeno avanço na saturação por água.

Figura 4 - a) Campo de Pressão com tempo de simulação de 600 dias. b) Perfil de Saturação com tempo de simulação de 600 dias.

a)

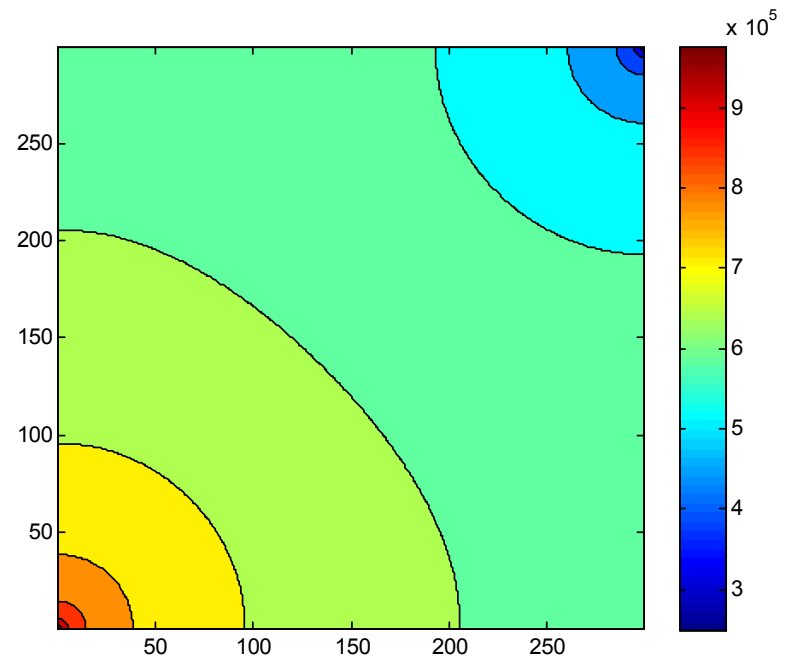

b)

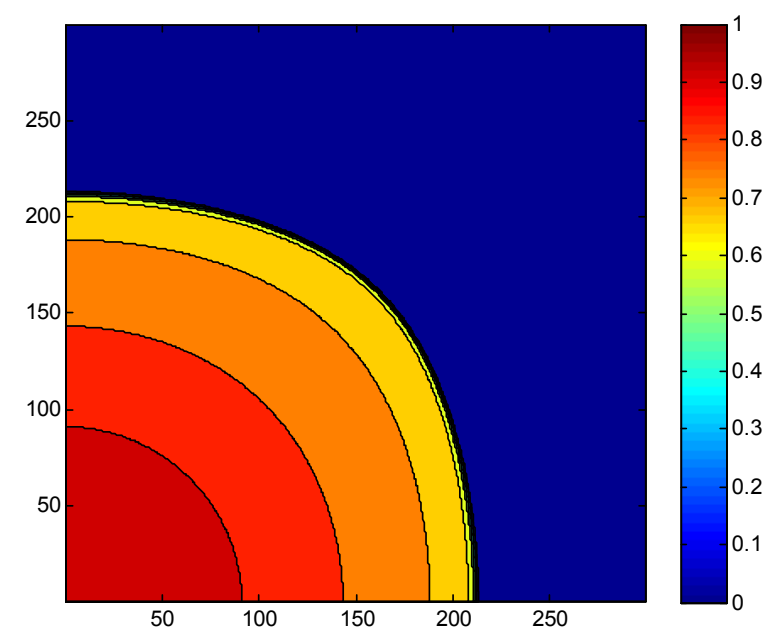

\section{CONCLUSAO}

Este artigo estudou a solução numérica do problema de escoamento bifásico em um reservatório de petróleo. Utilizando técnicas adequadas para cada tipo de equação, foi possível realizar a simulação da produção de óleo, com resultados consistentes que estão de acordo com a literatura.

\section{REFERÊNCIAS}

AARNES, J., GIMSE, T., LIE, K.A.: An introduction to the numerics of flow in porous media using Matlab. Springer Berlin Heidelberg, 2007.

CARVALHO, D. K. Uma Formulação do Método dos Volumes Finitos com Estrutura de Dados por Aresta para a Simulação de Escoamentos em Meios Porosos. Tese (Doutorado em Engenharia Civil) - UFPE, Recife, 2005.

CORDAZZO, J. Simulação de Reservatórios de Petróleo Utilizando o Método EbFVM e Multigrid Algébrico. Tese (Doutorado em Engenharia Mecânica) - UFSC, Florianópolis, 2006.

MALISKA, C. R. Transferência de Calor e Mecânica dos Fluidos Computacional. 2 $2^{\mathrm{a}}$. Edição. Rio de Janeiro: Livros Técnicos e Científicos Editora, 2004.

PEACEMAN, D. Fundamentals of Numerical Reservoir Simulation. Amsterdam: Elsevier, 1977. 